\title{
Multiple Exciton Coherence Sizes in Photosynthetic Antenna Complexes viewed by Pump-Probe Spectroscopy
}

\author{
T. Meier, V. Chernyak, and S. Mukamel* \\ Department of Chemistry and Rochester Theory Center for Optical Science and Engineering, \\ University of Rochester, Rochester, New York 14627
}

Received: December 20, $1996^{\otimes}$

\begin{abstract}
The pump-probe signal from the light-harvesting antenna LH2 of purple bacteria is analyzed using a Green function expression derived by solving the nonlinear exciton-oscillator equations of motion (NEE). A microscopic definition of the exciton mean free path $\left(L_{\mathrm{f}}\right)$ and localization size $\left(L_{\rho}\right)$ is given in terms of the off-diagonal elements of the exciton Green function and density matrix, respectively. Using phonon-induced (homogeneous) and disorder-induced (inhomogeneous) line widths compatible with superradiane measurements, we find that at $4.2 \mathrm{~K}$ the localization size is $L_{\rho}=15$ and that the shift $\Delta \Omega$ between the positive and negative peaks in the differential absorption is determined by a different effective size $L_{\mathrm{f}} / 2=5.6$ associated with the exciton mean free path. Our model further predicts the recently observed superradiance coherence size determined by $L_{\rho}$.
\end{abstract}

\section{Introduction}

Optical properties of photosynthetic antenna complexes have become recently an object of intensive experimental and theoretical studies. ${ }^{1,2}$ The $2.5 \AA$ A resolution geometrical structure of the peripheral antenna of purple bacteria $(\mathrm{LH} 2)^{3}$ shows 27 bacteriochlorophyll $a$ (BChla) monomers arranged in two rings: 9 weakly coupled $\mathrm{BChla}$ molecules form the outer ring which is responsible for the higher energetic B800 band, while 18 strongly interacting BChla of the inner ring form the lower energetic B850 band. This high circular symmetry makes it possible to estimate intermolecular interaction parameters and has triggered intensive theoretical modeling of optical signals including absorption,,${ }^{4,5}$ pump-probe, ${ }^{11-16}$ and three pulse echoes. ${ }^{8,17}$ These measurements show ultrafast energy transfer and vibrational coherence which persists even on time scales where electronic coherence has been lost. Similar observations have been made in the 32-member ring of the LH1 system..$^{10,17-20}$

At low temperatures $(4 \mathrm{~K})$ a broad absorption line $\Lambda \approx 300$ $\mathrm{cm}^{-1}$ (fwhm) is observed in LH2, whereas hole-burning studies report a $210 \mathrm{~cm}^{-1}$ hole which is independent of the burn frequency. ${ }^{10}$ At room temperature, polarization-dependent frequency-domain pump-probe studies give a homogeneous width varying between $200 \mathrm{~cm}^{-1}$ at the band center and $30 \mathrm{~cm}^{-1}$ at the red wing. ${ }^{21} \mathrm{~A}$ total line width of $\Lambda=430 \mathrm{~cm}^{-1}$ and a homogeneous width $188 \mathrm{~cm}^{-1}$ was found in ref $17 . \Lambda=300$ $\mathrm{cm}^{-1}$ was reported in ref 4 . The observed line width reflects the combined influence of static disorder (inhomogeneous broadening), and exciton coupling to intermolecular, intramolecular, and solvent nuclear motions (homogeneous line width). Coherent and incoherent excitonic dynamical processes are strongly affected by both broadening mechanisms. Pumpprobe spectroscopy provides a direct view into excitonic motions through the differential absorption of a probe pulse as a function of its frequency and the time delay with respect to a pump pulse. The frequency-dependent differential absorption typically contains a negative peak related to bleaching and to stimulated emission from the one-exciton band to the ground state (we refer to both contributions as BL), and a positive peak which reflects excited-state absorption (ESA) from one-exciton to two-

\footnotetext{
${ }^{\otimes}$ Abstract published in Advance ACS Abstracts, August 1, 1997.
}

exciton states. ${ }^{11,15}$ The shift $\Delta \Omega$ between these two features is usually attributed to the energy difference between the exciton state prepared by the pump, and a two-exciton state coupled to that exciton by the probe. ${ }^{22}$ In a linear aggregate made out of $L$ molecules we have $\Delta \Omega \approx 3 \pi^{2} J /(L+1)^{2}$, where $J$ is the nearest-neighbor exciton intermolecular interaction. The shift in the pump-probe signal of $J$ aggregates has been observed by Wiersma's group and fitted using a model of a linear aggregate of physical size $L=15$ with nearest-neighbor interaction $J=1227 \mathrm{~cm}^{-1}$ derived from the frequency shift between the aggregate and the monomer absorption peaks. ${ }^{23}$ This coherence size was associated with the Anderson localization length of excitons, induced by static disorder. ${ }^{24}$ Further numerical simulations of the signal in the presence of Gaussian energy (diagonal) disorder with a finite correlation length show excellent agreement with experiment. ${ }^{25}$ Similar observations have been made recently in both LH1 and LH2. ${ }^{11,15}$ For LH1, $\Delta \Omega$ increases monotonically with temperature from $24 \mathrm{~cm}^{-1}$ for $T=4 \mathrm{~K}$ to $440 \mathrm{~cm}^{-1}$ for $T=280 \mathrm{~K}$. For LH2 the temperature dependence is more complicated. ${ }^{15}$ From 4 to 20 $\mathrm{K}$ the shift decreases from 250 to $215 \mathrm{~cm}^{-1}$, and for higher temperatures it increases, reaching $280 \mathrm{~cm}^{-1}$ at room temperature..$^{15}$

In a one-dimensional circular aggregate with $L$ molecules and nearest-neighbor exciton coupling $J$ the single-exciton band has energies $2 J \cos (k), k=2 \pi n / L, n=0,1, \ldots, L-1$. The spacing between the levels at the band center is $4 \pi J / L$. By calculating the two-exciton states, it was shown ${ }^{22}$ that $\Delta \Omega=4 \pi^{2} J / L^{2}$ coincides with the spacing between the two lowest exciton states. The role of static disorder may be best understood using the Mott picture of localization ${ }^{26}$ according to which wave functions of excitons with close energies may not overlap. This is connected with the statistical property of level repulsion. ${ }^{27}$ This results in a fundamental characteristic energy $\Delta E$, namely, the minimal splitting between two overlapping localized states. Using this argument, one can divide the aggregate into spatially nonoverlapping segments whose sizes are determined by the Anderson localization length $l_{\mathrm{A}}$, and obtain for the energy splitting between two lowest excitons in a segment $\Delta E_{\mathrm{A}} \approx 3 \pi^{2} J /\left(l_{\mathrm{A}}+1\right)^{2}$. The pump-probe signal can then be interpreted using a three-level model which contains the ground 
state and the lowest one-exciton and two-exciton states in a linear aggregate with size $l_{\mathrm{A}}$. This argument relates the peak shift in the pump-probe signal directly to the exciton localization size and yields $\Delta \Omega \approx \Delta E_{\mathrm{A}}$. In the interpretation of recent pump-probe experiments, this shift has been used to estimate the size of coherently coupled subunits of LH2. ${ }^{11,15}$ To obtain shifts on the order of $200 \mathrm{~cm}^{-1}$ it had to be assumed that only a fraction of the monomers $L=2-4$ can be coherently coupled. Fitting of the pump-probe data using a model with different physical sizes ( $L=3$ or 4$)$ yields values for the coherence size for LH1 of 3.2 for $4 \mathrm{~K}$ and 2.3 for $280 \mathrm{~K}$, and for LH2 of 2.3 for $4 \mathrm{~K}$ and $\approx 2.7$ for $280 \mathrm{~K}^{15}$ It was not possible to reproduce the experimentally observed shifts by calculations which included the entire ring. Another recent measurement of cooperative spontaneous emission (superradiance), interpreted using disorder resulted in a coherence size of $2.8 .^{28}$

It is clear that a proper theory of the optical response of these aggregates should not be limited to the localization size and should take into account other coherence sizes as well. In this paper we identify various coherence sizes in LH2 and discuss their spectroscopic signatures using a microscopic model that takes into account the aggregate structure as well as effects of static disorder and exciton-phonon coupling. The exciton mean free path $L_{\mathrm{f}}$ represents the exciton-dephasing length scale. Its origin can be understood as follows: In the presence of static disorder or coupling with phonons the exciton wave vector is no longer a good quantum number, and the exciton eigenstates become wavepackets in $k$ space with width $\Delta k_{\mathrm{f}} \approx 2 \pi / L_{\mathrm{f}}$, where $L_{\mathrm{f}}$ is the mean free path which reflects exciton dephasing. In the vicinity of the band-edge the width $\Delta k_{\mathrm{f}}$ induces a width $\Gamma_{\mathrm{f}}$ $=2 J\left(1-\cos \left(\Delta k_{\mathrm{f}}\right)\right)$ in energy space (assuming nearest-neighbor coupling $J$ ). This gives $\Lambda_{\mathrm{f}}=2 \Gamma_{\mathrm{f}} \approx 8 \pi^{2} J / L_{\mathrm{f}}{ }^{2}$ for the linear absorption line width. In the case of static disorder exciton dephasing is controlled by elastic scattering and $L_{\mathrm{f}}=l_{\mathrm{d}}$, where $l_{\mathrm{d}}$ is the disorder-induced mean free path, which represents the inhomogeneous line width $\Lambda_{\mathrm{d}}$. Exciton-phonon coupling introduces an additional contribution to the mean free path. In this case the total (exciton and phonon) momentum is a good quantum number, which again introduces a width $\Delta k$ into the purely excitonic momentum, determined by the phonon-induced mean free path $l_{\mathrm{ph}}$. This results in a homogeneous line width of $\Lambda_{\mathrm{ph}} \approx 8 \pi^{2} J / l_{\mathrm{ph}}{ }^{2}$.

In one-dimensional systems with weak static disorder the localization length $l_{\mathrm{A}}$ coincides with the mean free path $l_{\mathrm{d}}, l_{\mathrm{d}}=$ $l_{\mathrm{A}}$. This is no longer the case once coupling with phonons is incorporated. Exciton-phonon scattering together with static disorder form a combined mean-free path $L_{\mathrm{f}}$ which is related to the line width $\Lambda_{\mathrm{f}}$ by $\Lambda_{\mathrm{f}} \approx 8 \pi^{2} J / L_{\mathrm{f}}^{2}$, and the total mean-free path $L_{\mathrm{f}}$ is in general different from the localization length $l_{\mathrm{A}}$.

The inelastic exciton mean free path $l_{\mathrm{ph}}$ can be larger or smaller than the localization length $l_{\mathrm{A}}$. When $l_{\mathrm{A}} \ll l_{\mathrm{ph}}$, we can treat the exciton-phonon interaction as a small correction to disorder. For $l_{\mathrm{A}} \approx l_{\mathrm{ph}}$, we have a combined effect of disorder and exciton-phonon coupling, and when $l_{\mathrm{ph}}<l_{\mathrm{A}}$, inelastic scattering destroys the effects of exciton localization. This is not the end of the story yet, since other coherence sizes enter as well. At finite temperatures an additional coherence size $l_{\mathrm{T}}$ $\approx 3 \pi^{2} J / k T$ appears whereby the temperature is the relevant energetic parameter (see Figure 7). For strong exciton-phonon coupling one needs to incorporate the polaron size $l_{\mathrm{p}}$ (polaron is an exciton dressed with a phonon cloud), with the polaron binding energy $E_{\mathrm{p}}$ being the corresponding energetic parameter.

The present analysis is based on a Green function theory of nonlinear spectroscopy developed in ref 29 and summarized in section II. This theory, in contrast to the sum over states (SOS)

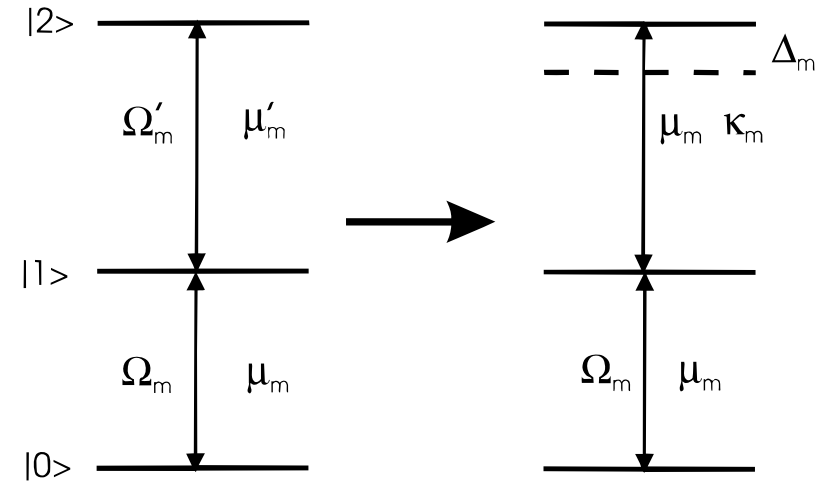

Figure 1. Mapping of the three-level model (left) onto the excitonic oscillator model (right). $\Delta_{m}=\Omega^{\prime}{ }_{m}-\Omega_{m}$ is an anharmonicity parameter. $^{31}$

approach, ${ }^{30}$ relates the optical signals to time-dependent exciton populations and coherences, exciton dephasing, and excitonexciton scattering using one-exciton states as an input and avoiding the explicit calculation of two-exciton states. The advantages of this formulation will be discussed in section II. In section III we present numerical calculations for the absorption and pump-probe spectra of $\mathrm{LH} 2$, including both elastic (disorder-induced) and inelastic (exciton-phonon) scattering contributions to exciton dephasing. In section IV we define the exciton mean free path by using the antidiagonal section of the exciton Green function. We show that the pump-probe peak shift $\Delta \Omega$ is primarily determined by $L_{\mathrm{f}}$ and obtain $L_{\mathrm{f}} \approx$ 11.2.

In the last two sections we show that at low temperatures the influence of exciton dephasing and localization on radiative decay rate is very different. We thus propose time-resolved fluorescence measurements to distinguish between effects of exciton dephasing and Anderson localization. In section $\mathrm{V}$ we introduce the exciton density matrix localization size $L_{\rho}$ based on its "antidiagonal" section which contains all relevant information about time-resolved fluorescence and allows us to visualize the exciton coherence size. We obtain $L_{\rho} \approx 15$. Our results are compatible with the relaxed fluorescence line shape, since the disorder-induced Stokes shift is smaller than the experimentally measured total Stokes shift. In section VI we show how $L_{\rho}$ controls the superradiance and predicts the correct superradiance size $L_{\mathrm{S}}$, in agreement with recent experiments.

\section{Doorway/Window Picture of Pump-Probe Spectroscopy}

We consider an aggregate made of $L$ three-level molecules with states $S_{0}, S_{1}$, and $S_{2}$ and energies $0, \Omega_{m}$, and $\Omega_{m}+\Omega^{\prime}{ }_{m}$, $m=1, \ldots, L$. The transition dipoles for the $S_{0} \rightarrow S_{1}$ and $S_{1} \rightarrow$ $S_{2}$ transitions will be denoted $\mu_{m}$ and $\mu_{m}^{\prime}$, respectively (see Figure 1). We assume that these are the only nonvanishing matrix elements of the dipole operator. This model does adequately represent the pump-probe spectroscopy of antenna complexes. ${ }^{11}$ To develop a collective approach to optical excitations in this system, we associate with each molecule an anharmonic oscillator degree of freedom with fundamental frequency $\Omega_{n}$ and creation (annihilation) operators $B^{\dagger}{ }_{n}\left(B_{n}\right)$. These operators satisfy the commutation relations:

$$
\left[B_{m}, B_{n}^{\dagger}\right]=\delta_{m n}\left(1-\left(2-\kappa_{m}^{2}\right) B_{m}^{\dagger} B_{m}\right)
$$

The parameter $\kappa_{m} \equiv \mu_{m}^{\prime} / \mu_{m}$ is the ratio of the two transition dipoles. We further introduce the parameter 
$g_{n} \equiv 2 \hbar\left(\Omega^{\prime}{ }_{n} \kappa_{n}{ }^{-2}-\Omega_{n}\right)$. In ref 31 it has been shown that the original model of coupled three-level systems with parameters $\left(\Omega_{m}, \Omega_{m}^{\prime}, \mu_{n}, \mu_{m}^{\prime}\right)$ can be mapped into coupled anharmonic oscillators characterized by the parameters $\Omega_{m}, \kappa_{m}$, and $g_{m}$.

The linear absorption spectrum has the form ${ }^{29}$

$$
\alpha(\omega)=\sum_{m n} \operatorname{Im}\left[\mu_{m} \mu_{n} G_{m n}(\omega)\right]
$$

Here

$$
G_{m n}(\omega)=\int_{0}^{\infty} \mathrm{d} \tau \exp (i \omega \tau)\left\langle B_{m}(\tau) B_{n}^{\dagger}(0)\right\rangle
$$

is the one-exciton Green function, where the time evolution of the $B_{m}(\tau)$ operator is determined by the free material Hamiltonian (without the fields). For our model, this Green function is given by $G_{m n}(\omega) \equiv\left[(\omega-h+i \Gamma)^{-1}\right]_{m n}$ with $h_{m n} \equiv \Omega_{m} \delta_{m n}$ $+\left(1-\delta_{m n}\right) J_{m n}$ is the one-exciton Hamiltonian and $\Gamma_{m n}$ is the one-exciton relaxation matrix which can be expressed in terms of bath spectral densities.

The calculation of the pump-probe signal $S(\omega ; \tau)$ as a function of the time delay $\tau$ and the probe frequency $\omega$ is simplified considerably when the following conditions hold: (i) the exciton population-relaxation time scale $\tau_{\mathrm{p}}$ is long compared with the inverse absorption line width $1 /(2 \Gamma)^{-1}$, (ii) the pump pulse is short compared to $\tau_{\mathrm{p}}$, (iii) the delay between the pump and probe is long compared to the duration of the pump pulse, (iv) the probe pulse is short compared to $\tau$ and long compared to $1 /(2 \Gamma)^{-1}$. These conditions define the "snapshot limit" 32 where the signal depends only on the pump and the probe frequencies and their relative delay and is independent on their envelopes. Applying the results of ref 29, we express the exciton Green function in terms of the exciton density matrix $\mathrm{N}_{m n}(t) \equiv\left\langle\tilde{B}_{m}^{\dagger}(t) \tilde{B}_{n}(t)\right\rangle$ (here the time evolution of the operators $\tilde{B}^{\dagger}{ }_{m}(t)$ and $\tilde{B}_{n}(t)$ is in the presence of the pump). By invoking the Kadanoff-Baym ansatz ${ }^{33,34}$ and combining it with the exciton scattering matrix $\bar{\Gamma}(\omega)$ derived in ref 31 we obtain for $\tau \gg \Gamma^{-1}$

$$
S(\omega, \tau)=\operatorname{Im} \sum_{m n} M_{m n}(\omega) N_{m n}(\tau)
$$

The signal (eq 4) has the following interpretation: two interactions with the pump create the reduced exciton density matrix $N_{m n}$ (the doorway) at $t=0$, which evolves in time for the delay period $\tau$, resulting in $N_{m n}(\tau)$. The diagonal elements $N_{n m}(\tau)$ represent exciton populations whereas the off diagonal elements $n \neq m$ are excited-state coherences. $N_{m n}(\tau)$, which can be calculated using various methods, ${ }^{35}$ plays a crucial role in time- and frequency-resolved fluorescence and energy transfer. The signal $S$ is related to the exciton density matrix by its Liouville space overlap with the window function $M_{m n}(\omega)$, given by

$$
M_{m n}(\omega) \equiv \sum_{i j l} \mu_{i} \mu_{j} G_{j l}(\omega) G_{m i}(\omega) T_{m n l}(\omega)
$$

with

$$
T_{m n l}(\omega) \equiv \int_{-\infty}^{\infty} \frac{\mathrm{d} \epsilon}{2 \pi i} G_{l n}^{*}(\epsilon) \bar{\Gamma}_{l m}(\omega+\epsilon)
$$

The two-exciton scattering matrix has the form

$$
\bar{\Gamma}_{m n}(\omega)=[F(\omega)]_{m n}{ }^{-1}\left[\left(\omega+g_{n}\right) \kappa_{n}^{2}-2 \omega\right]
$$

with

$$
\begin{aligned}
\bar{\Gamma}_{m n}(\omega)=\delta_{m n} \kappa_{m}{ }^{2}-\left[\left(\omega+g_{n}\right) \kappa_{n}{ }^{2}-2 \omega\right] \mathrm{G}_{m n}(\omega)+ \\
2 i \kappa_{m}{ }^{2} \sum_{k} \mathrm{R}{ }_{m k} \mathrm{G}_{k n}(\omega)
\end{aligned}
$$

$$
\mathrm{G}_{m n}(\omega) \equiv \int_{-\infty}^{\infty} \frac{\mathrm{d} \epsilon}{2 \pi i} G_{m n}(\epsilon) G_{m n}(\omega-\epsilon)
$$

$\mathrm{R}_{m k}$ is the relaxation operator of the third level $\left(S_{2}\right)$.

The time- and frequency-resolved fluorescence signal $S_{\mathrm{fl}}(\omega, \tau)$ is also given by eq 4 by simply replacing $M$ with the fluorescence window function $M^{(\mathrm{fl})}(\omega)$

$$
M_{m n}^{(\mathrm{fl})}(\omega)=\sum_{l} \mu_{l} \mu_{n} G_{l m}(\omega)
$$

This doorway-window picture has been applied earlier to describe nuclear dynamics in nonlinear spectroscopy of a single chromophore. $^{32}$ The present theory extends it to excitonic dynamics and allows us to separate the optical process into preparation, evolution, and detection states, and analyze separately the roles of the pump (which determines the doorway) and the probe (which affects the window). The conventional SOS approach relates optical signals to properties of individual eigenstates. An exciton state created by the pump is coupled by the probe to a continuous manifold of two-exciton states (in the joint exciton-phonon space). The signal is determined by the distribution of the dipole operator matrix elements between one- and two-exciton states; the necessary statistical (collective) properties of levels can be described using multidimensional spectral densities. ${ }^{36}$ The Green function approach offers a very different and a much simpler physical picture for the optical response in terms of exciton-exciton scattering and has numerous advantages compared with the SOS: (i) it does not suffer from the problem of cancellation of terms which scale as $\sim L^{2}$ in nonlinear optical signals. ${ }^{22}$ These cancellations often create severe numerical difficulties and make it hard to gain physical insight. (ii) The diagonalizations of $L^{2} \times L^{2}$ matrixes necessary for calculating two-exciton states are completely avoided. Only $L \times L$ matrixes need to be inverted, which considerably reduces the numerical effort. The most time consuming step in calculating the pump-probe signal is the multiple summations which appear in eqs 4 and 5 and not the matrix inversion. (iii) Exciton-phonon interactions are naturally incorporated.

In ref 29 these Green functions expressions have been derived using diagrammatic techniques. A much more intuitive derivation based on the non-linear exciton equations of motion (NEE) was developed recently ${ }^{37}$ and will be briefly sketched below. In general, for our model, any third order optical process (such as pump-probe) may be calculated by following the time evolution of the variables $\left\langle B_{n}\right\rangle,\left\langle B_{n} B_{m}\right\rangle,\left\langle B_{n}^{\dagger} B_{m}\right\rangle$, and $\left\langle B_{n}^{\dagger} B_{m} B_{l}\right\rangle$. Equations 4-9 can be derived using equations of motion for these variables, which may be closed using projection operator techniques. ${ }^{38}$ The microscopic description of the pump-probe spectrum depends on three time intervals. The first is the difference between the two interactions with the pump. During this interval the variables $\left\langle B_{n}\right\rangle$ are created, and the doorway is prepared. Following the second interaction with the pump we start the second delay period $\tau$, where the density matrix $\left\langle B_{n}^{\dagger} B_{m}\right\rangle$ evolves in time. Here an explicit relaxation kernal obtained, 
e.g., using the Redfield theory ${ }^{37,39}$ should be used. For long time delays $\tau$, the exciton density matrix $N_{m n}(\tau)$ assumes the thermally equilibrated form

$$
N_{m n}=Z^{-1} \sum_{\alpha} \Psi_{\alpha}^{*}(n) \Psi_{\alpha}(m) \exp \left(-\epsilon_{\alpha} / T\right)
$$

where $\Psi_{\alpha}$ and $\epsilon_{\alpha}$ are the exciton wave functions and energies, and $Z \equiv \sum_{\alpha} \exp \left(-\epsilon_{\alpha} / T\right)$.

Subsequently, the first interaction with the probe creates the three-exciton variable $\left\langle B_{n}^{\dagger} B_{m} B_{l}\right\rangle$ reflecting coherence between one- and two-exciton states, as well as $\left\langle B_{n}\right\rangle$, whose time evolution during the third evolution period between the two interactions with the probe determines the window. The major difficulty in implementing this procedure is the proper incorporation of the $\left\langle B_{n}^{\dagger} B_{m} B_{l}\right\rangle$ variables since their number scales as $\sim L^{3}$. Various factorization schemes have been employed in the past to describe specific measurements. A $\left\langle B_{n}^{\dagger}\right\rangle\left\langle B_{m} B_{l}\right\rangle$ factorization $^{22}$ applies in the absence of phonons. A $\left\langle B_{n}^{\dagger} B_{m}\right\rangle$ $\left\langle B_{l}\right\rangle$ factorization was used to describe exciton transport. ${ }^{40,41} \mathrm{~A}$ more elaborate maximum entropy factorization which interpolates between the above two was used in the analysis of offresonant optical susceptibilities. ${ }^{32,42}$ The NEE derived recently uses an approximate relaxation kernel for the $\left\langle B_{n}^{\dagger} B_{m} B_{l}\right\rangle$ variables rather than factorizing these variables themselves. Phonon exchange among different excitons is neglected. This means that during the third time interval phonon effects are incorporated through the one-exciton lifetime induced by excitonphonon dephasing, whereas exciton-exciton scattering is taken into account explicitly. This scattering is related to Pauli exclusion and to anharmonicities induced by the third molecular level.

Equations 4-9 may be used to analyze the combined effects of disorder and coupling with phonons on the pump-probe signal. Previous calculations have employed a model with disorder alone where exciton localization is the only size underlying $\Delta \Omega .{ }^{11,15}$ In that case, the BL peak comes from the resonant feature in $\operatorname{Im}\left[G_{n s}(\omega) G_{i m}(\omega)\right]$ while the ESA peak is due to a sharp resonance in $\operatorname{Im}[\bar{\Gamma}(\omega)]$ which enters the signal through eqs 4 and 5. One can then qualitatively describe the system as an ensemble of three-level systems. Once the phononinduced homogeneous width becomes large compared to the spacing between two-exciton levels, the resonances of $\bar{\Gamma}$ overlap and the pump-probe signal assumes a very different nature: it reflects a collective (global) excitation of the entire two-exciton manifold. A key question is whether $\Delta \Omega$ originates from exciton localization or from electronic dephasing determined by the parameter $L_{\mathrm{f}}$. The calculations presented in the coming sections will address this issue.

\section{Application to the B850 System of LH2}

We now apply the doorway-window expressions to calculate the pump-probe signals from the B850 band of LH2. This band comes from a cyclic aggregate consisting of 18 chlorophyll molecules. We neglect the weak coupling to the outer ninemolecule ring (the B800 band), and each chlorophyll molecule is modeled as a three-level system. ${ }^{11,15,31}$ The transition frequency between the second and third levels is shifted to the blue compared with the lowest transition, and we set the anharmonicity of the third level $\Omega^{\prime}{ }_{m}-\Omega_{m}=100 \mathrm{~cm}^{-1}$. ${ }^{11}$ The ratio of the dipole moments is assumed to be the same for all molecules and was adjusted to fit the experimental pump-probe spectra. This results in $\kappa$ values between $\approx 0.9$ and 1.0 . We used the molecular positions measured by McDermott et al. ${ }^{3}$ The system is made of nine dimers, and we assume that the

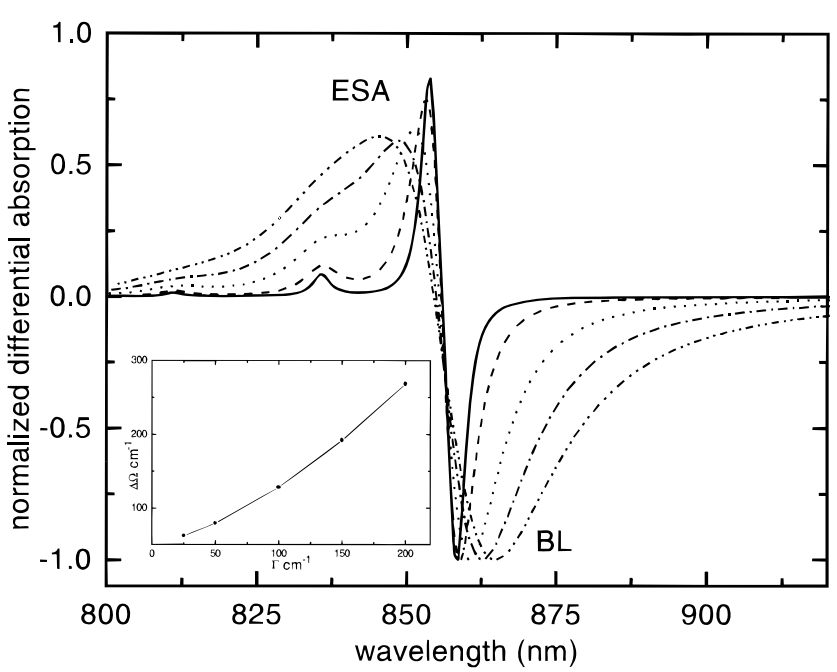

Figure 2. Normalized pump-probe spectra without disorder for different dephasing rates $\Gamma$ at 4.2 K. $\kappa=0.9$. Solid $\Gamma=25 \mathrm{~cm}^{-1}$; dash $50 \mathrm{~cm}^{-1}$; dot $100 \mathrm{~cm}^{-1}$; dash-dot $150 \mathrm{~cm}^{-1}$; and dash-dot-dot 200 $\mathrm{cm}^{-1}$. Inset: shift $\Delta \Omega$ as a function of $\Gamma$. For other parameters see text.

transition dipoles of individual molecules are tangential to the ring and are almost antiparallel within each dimer. ${ }^{3,4}$ The excitonic couplings between the molecules were taken from Sauer et al.. ${ }^{4}$ nearest-neighbor couplings of 273 and $291 \mathrm{~cm}^{-1}$, next-nearest-neighbor coupling of -50 and $-36 \mathrm{~cm}^{-1}$, and the third-nearest-neighbor term of $12 \mathrm{~cm}^{-1}$. All other couplings were reported to be smaller than $10 \mathrm{~cm}^{-1}$ and were therefore neglected in the present calculation. [Although the nearestneighbor coupling constants in the B850 ring on LH2 are positive, spectroscopic features of the system are similar to those of $J$ aggregates (e.g., the aggregate absorption band is red-shifted compared to the monomer) due to almost antiparallel directions of nearest-neighbor dipoles. A simple transformation $B_{n} \rightarrow$ $(-1)^{n} B_{n}$ leads to a representation where the nearest-neighbor coupling constants are negative and the dipoles are almost parallel.]

In the absence of specific information we assumed the simplest model for the relaxation matrices $\Gamma_{m n}$ and $\mathrm{R}{ }_{m n}$. These matrixes were taken to be diagonal in the site representation with all diagonal elements equal $\Gamma_{m n}=\delta_{m n} \Gamma, \mathrm{R}_{m n}=\delta_{m n} \Gamma$; the homogeneous contribution to the linear absorption line width results in fwhm of $\Lambda=2 \Gamma$. Note that for this model the relaxation matrixes are also diagonal in the exciton representation with all relaxation rates being equal. The entire excitonphonon coupling is thus represented by a single parameter.

Previous modeling of the pump-probe spectra included disorder but neglected exciton-phonon coupling. ${ }^{11,15}$ We start our analysis by considering whether the reverse assumption, namely, exciton-phonon coupling (homogeneous broadening) alone with no disorder can reproduce the same spectra and lead to large $\Delta \Omega$, as observed experimentally. ${ }^{11,15}$

Figure 2 shows calculated pump-probe spectra without disorder for different phonon-induced homogeneous width $\Gamma$ at $4.2 \mathrm{~K}$. For all values of $\Gamma$ the pump-probe signals show a negative BL part at lower energies and a positive ESA part at higher energies. For small $\Gamma$, the splitting $\Delta \Omega$ is small, which indicated that this system has two-exciton states energetically very close to twice the one-exciton energy. In this case the ESA shows a progression of several well-resolved two-exciton contributions. As $\Gamma$ is increased, the contributions of different two-exciton states merge and $\Delta \Omega$ increases (see inset in Figure $2)$. In this regime the shift does not reflect an energetic difference between specific states but is induced by, and scales 

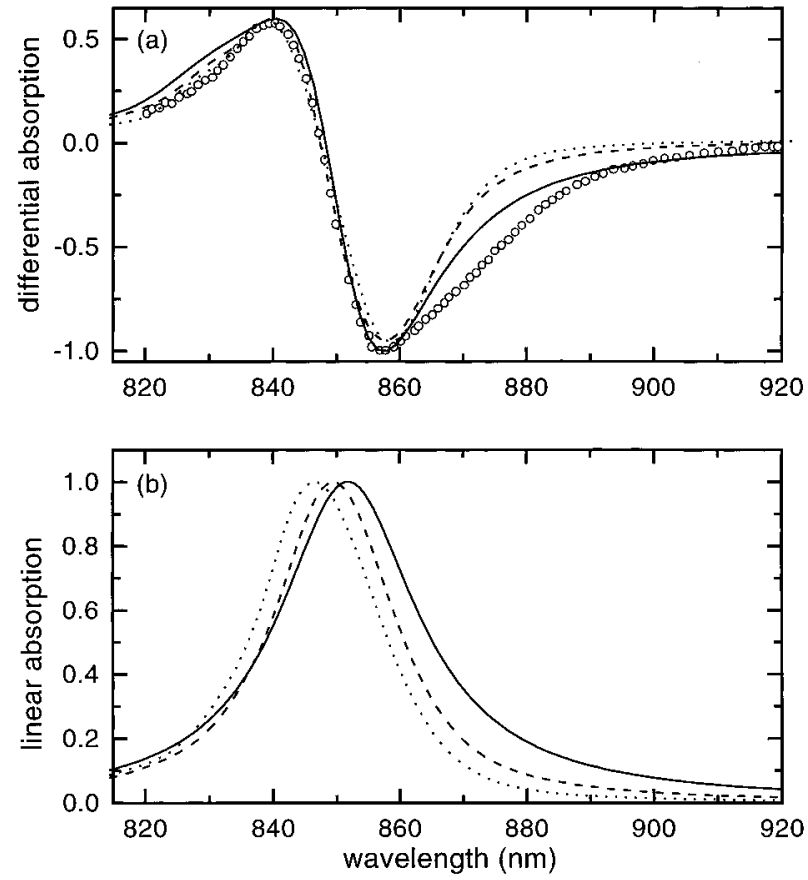

Figure 3. Normalized pump-probe (a) and linear absorption spectra (b) for models I (solid), II (dashed), and III (dotted). I $\Gamma=180 \mathrm{~cm}^{-1}$, $\sigma=0, \Omega_{m}=12348 \mathrm{~cm}^{-1}$, and $\kappa=0.9$; II $\Gamma=100 \mathrm{~cm}^{-1}, \sigma=377$ $\mathrm{cm}^{-1}, \Omega_{m}=12408 \mathrm{~cm}^{-1}$, and $\kappa=0.9$; III $\Gamma=50 \mathrm{~cm}^{-1}, \sigma=527$ $\mathrm{cm}^{-1}, \Omega_{m}=12488 \mathrm{~cm}^{-1}$ and $\kappa=1.0$; at $T=4.2 \mathrm{~K}$. The experimental data (circles) are taken from ref 15 .

with, the homogeneous line width. These calculations show that both disorder-induced localization and homogeneous broadening induced by coupling to phonons may account for the observed spectra.

We next calculated the pump-probe signal, incorporating both exciton-phonon interactions and static disorder, and fitted the shift between the BL and ESA as well as their relative magnitude to recently measured pump-probe spectra on B850 of LH2 at $4.2 \mathrm{~K}^{15}$ The exciton-phonon coupling strength can be obtained from hole-burning measurements, ${ }^{10}$ polarizationdependent frequency-domain pump-probe, ${ }^{21}$ and three-pulse echo measurements. ${ }^{17}$ Disorder is incorporated by assuming that each frequency $\Omega_{m}$ has a Gaussian distribution with fwhm $\sigma$ around its mean value. This model is known as Gaussian diagonal disorder. $\sigma$ was varied to fit the experimental pumpprobe spectra. Calculations were made using a Monte Carlo sampling over different realizations of disorder. In the following calculations we used three models. In model I we took $\Gamma=$ $180 \mathrm{~cm}^{-1}, \kappa=0.9, \Omega_{m}=12348 \mathrm{~cm}^{-1}$, and $\sigma=0$. In this case we neglected disorder altogether, and the absorption line width is homogeneous. In model II we have $\Gamma=100 \mathrm{~cm}^{-1}, \kappa$ $=0.9, \Omega_{m}=12408 \mathrm{~cm}^{-1}$, and $\sigma=377 \mathrm{~cm}^{-1}$, and finally in model III $\Gamma=50 \mathrm{~cm}^{-1}, \kappa=1.0, \Omega_{m}=12488 \mathrm{~cm}^{-1}$, and $\sigma=$ $527 \mathrm{~cm}^{-1}$. Considering previous measurements ${ }^{10,17,21}$ and estimates of the homogeneous and inhomogeneous line widths in LH2, model III is probably the most realistic.

Figure 3 compares the pump-probe and the linear absorption spectra for the three models at $4.2 \mathrm{~K}$. The linear absorption line width is $360 \mathrm{~cm}^{-1}$ (fwhm) for model I and $310 \mathrm{~cm}^{-1}$ for models II and III, which fall within the range of experimentally reported values.

The calculated pump - probe signals have been normalized, and $\Omega_{m}$ has been shifted so that the maximum of the BL is -1 and occurs at $858 \mathrm{~nm}$; these spectral shifts are 88, 148, and $228 \mathrm{~cm}^{-1}$ for models I, II, and III, respectively. To fit the linear spectrum, Sauer et al. ${ }^{4}$ have lowered the transition frequencies

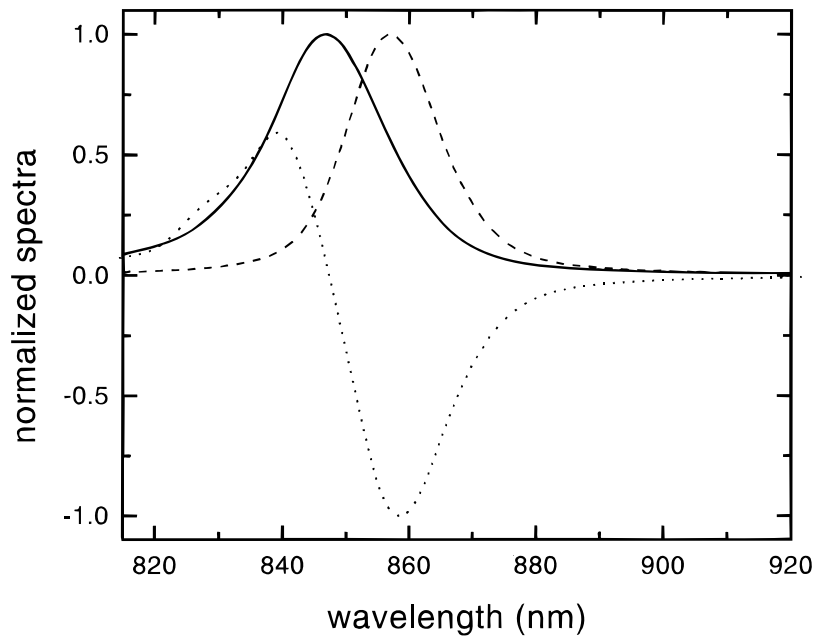

Figure 4. Linear absorption (solid), fluorescence (dashed), and pumpprobe (dotted) spectra calculated using model III.

of the B850 monomers by $240 \mathrm{~cm}^{-1}$ compared to the B800 monomers. The shifts obtained here are in the opposite direction and should reduce the difference between the monomers of the two bands. All three models yield about the same $\Delta \Omega$ and the same ratio of the magnitudes of BL and ESA. This demonstrates that the pump-probe experiment alone can be interpreted using very different models.

In Figure 4 we display the linear absorption and the relaxed fluorescence for model III. In the calculation of the fluorescence we have assumed that for each realization of disorder the excitons are in thermal equilibrium, which for the temperature of $4.2 \mathrm{~K}$ implies that only the lowest exciton state on each ring is occupied. The purely electronic disorder-induced Stokes shift shown in Figure 4 is $139 \mathrm{~cm}^{-1}$. In ref 17 the Stokes shift induced by nuclear motion at room temperature has been estimated to be about $80 \mathrm{~cm}^{-1}$. Since the disorder-induced Stokes shift should decrease with increasing temperature, these values are in good agreement with ref 43 , where the total Stokes shift has been measured to vary from $400 \mathrm{~cm}^{-1}$ at $4 \mathrm{~K}$ to 270 $\mathrm{cm}^{-1}$ at room temperature by comparing absorption and fluorescence. Thus using three parameters: $\Gamma_{\mathrm{ph}}$ related to the value of the exciton-phonon coupling, the strength of static disorder $\sigma$, and the ratio of the molecular dipoles $\kappa$, we obtain a good agreement between our calculations and four types of optical measurements: absorption, fluorescence and pumpprobe.

It should be noted that polaron effects are not included in the present theory. This means that the Stokes shift clearly seen in Figure 4 is not related to nuclear relaxation and reflects pure electronic relaxation. It will be interesting to explore whether the difference between the theoretical curves and experiment on the red wing of the pump- probe signal, ${ }^{15}$ could be attributed to polaron effects. ${ }^{15}$

\section{Exciton Mean Free Path and the Pump-Probe Peak Shift}

The exciton-phonon coupling and the static disorder parameters used in model III reproduce the pump-probe signal ${ }^{11,15}$ as well as linear absorption, ${ }^{4}$ of the B850 system of LH2. It is tempting to try to interpret these measurements in terms of some effective aggregate size $L_{\text {eff. }}$ The idea is that the combined effects of disorder and coupling with phonons restrict the extent of spatial coherence in the system. The optical properties should then depend on $L_{\text {eff }}$ rather than on the physical size of the aggregate (which for very large aggregates becomes irrelevant). 
One obvious candidate for $L_{\mathrm{eff}}$ is the localization size $l_{\mathrm{A}}$. If $l_{\mathrm{A}}$ controls the pump-probe signal the shift $\Delta \Omega$ reflects properties of individual exciton states. A different length scale which could determine $L_{\mathrm{eff}}$ is the mean free path $L_{\mathrm{f}}$. the formal definition of $L_{\mathrm{f}}$ involves the one-exciton Green function $G_{m n^{-}}$ $(\omega)$ introduced in section II: $L_{f}$ is a length scale of decay of $G_{m n}(\epsilon)$ as a function of $m-n, \epsilon$ being the exciton energy, chosen to be at the absorption maximum since these excitons primarily determine the optical response. We shall define $L_{\mathrm{f}}$ as the inverse participation ratio ${ }^{44}$ associated with $G_{m n}(\epsilon)$ :

$$
L_{\mathrm{f}} \equiv\left[L \sum_{m n}\left|G_{m n}(\epsilon)\right|^{2}\right]^{-1}\left[\left(\sum_{m n}(\epsilon) \mid\right)^{2}\right]
$$

This quantity gives the length scale on which the exciton Green function decays along the "antidiagonal" direction, i.e., as a function of $n-m$.

$\left|G_{m n}(\epsilon)\right|$ for models I, II, and III displayed in Figure $5 \mathrm{~d}-\mathrm{f}$ shows how they decay with increasing $|m-n|$. The mean free paths $L_{\mathrm{f}}$ calculated using eq 15 assume the values 9.2, 10.6, and 11.2, respectively. To show how the mean free path enters into the pump-probe signal, we shall carry out the integration in eq 6 using the exciton basis set and switch to the exciton representation for the Green functions. Since after averaging over disorder the translational symmetry of a circular aggregate is restored, and denoting the momentum of the $\alpha$ th exciton by $k_{\alpha}$ we obtain for the signal

$$
\begin{aligned}
& S(\omega, \tau)= \\
& \sum_{\alpha} N_{\alpha \alpha}(\tau) \operatorname{Im}\left[G^{2}(\omega, k)\right] \operatorname{Re}\left[\bar{\Gamma}\left(\omega+\epsilon_{\alpha}+i \Gamma, k+k_{\alpha}\right)\right] \\
& \sum_{\alpha} N_{\alpha \alpha}(\tau) \operatorname{Re}\left[G^{2}(\omega, k)\right] \operatorname{Im}\left[\bar{\Gamma}\left(\omega+\epsilon_{\alpha}+i \Gamma, k+k_{\alpha}\right)\right]
\end{aligned}
$$

where $N_{\alpha \alpha}(\tau)$ are exciton populations at time $\tau$, and $k=2 \pi / L$ is the momentum of the optically active exciton. When the exciton dephasing rate $\Gamma$ is larger than the splitting between exciton levels, the two-exciton resonances merge and $\bar{\Gamma}(\omega+$ $\left.\epsilon_{\alpha}+i \Gamma, k+k_{\alpha}\right)$ in the right-hand side of eq 13, shows no sharp resonances. (However, its imaginary part when $\omega+\epsilon_{\alpha}$ is tuned across the two-exciton band is a signature of twoexciton states.) The resonances of the pump-probe signal are thus given by the Green functions $G$. The shift $\Delta \Omega$ now reflects collective properties of the two-exciton manifold rather than positions of individual two-exciton states. Under these circumstances, the pump-probe signal may not be interpreted in terms of a three-level model. Instead, the signal is related to the coupled dynamics of exciton populations and coherences, exciton dephasing, and exciton-exciton scattering, as represented by eqs $4-9$. This is how the mean free path (which is related to the spatial coherence of the Green function) enters into the picture.

Since $G(\omega)$ has sharp one-exciton resonances, $\operatorname{Im}\left[G^{2}(\omega)\right]$ has an absorptive negative profile while $\operatorname{Re}\left[G^{2}(\omega)\right]$ is dispersive. The sum of these two terms gives the positive and negative peaks. The former originates from the positive feature in the dispersive form of $\operatorname{Re}\left[G^{2}(\omega)\right]$ and is proportional to $\operatorname{Im}\left[\bar{\Gamma}\left(\omega+\epsilon_{\alpha}+i \Gamma\right)\right]$ which is nonzero by virtue of the twoexciton manifold. The shift between the peaks is determined by the shift between the positive and negative features of $\operatorname{Re}\left[G^{2}(\omega)\right]$ which yields $\Delta \Omega \approx 2 \Gamma / \sqrt{3}$. In this qualitative picture we neglected the weak frequency dependence of $\bar{\Gamma}\left(\omega+\epsilon_{\alpha}+i \Gamma\right)$. This rough estimate is verified by our numerical calculations: using the absorption line width $2 \Gamma=$ $310 \mathrm{~cm}^{-1}$ (models II and III) we obtain $\Delta \Omega=180 \mathrm{~cm}^{-1}$ versus $\Delta \Omega=250 \mathrm{~cm}^{-1}$ obtained in our numerical calculations. For model I (no disorder) we have even a better agreement: $\Delta \Omega=$ $210 \mathrm{~cm}^{-1}$ (for $2 \Gamma=360 \mathrm{~cm}^{-1}$ ) versus $250 \mathrm{~cm}^{-1}$.

We are now in a position to relate the shift $\Delta \Omega$ in the pumpprobe signal to the exciton mean free path $L_{\mathrm{f}}$. We note that the signal (eq 13) depends on $\operatorname{Im}\left[G_{m n}{ }^{2}(\omega)\right]$ and $\operatorname{Re}\left[G_{m n}{ }^{2}(\omega)\right]$. We shall define three new length scales $L_{\mathrm{f}}^{(i)}, L_{\mathrm{f}}^{(r)}$, and $L_{\mathrm{f}}^{(a)}$, obtained from eq 12 by substituting $\rho_{m n}^{(i)}=\operatorname{Im}\left[G_{m n}{ }^{2}(\epsilon)\right], \rho_{m n}^{(r)}=\operatorname{Re}\left[G_{m n}{ }^{2}-\right.$ $(\epsilon)]$, and $\rho_{m n}^{(a)}=\left|G_{m n}{ }^{2}(\epsilon)\right|$ for $G_{m n}(\epsilon)$, respectively. These reflect how the imaginary part, the real part, and the absolute value of $G^{2}$ decay with $|n-m|$, respectively $\rho_{m n}^{(r)}, \rho_{m n}^{(i)}$, and $\rho_{m n}^{(a)}$ for model III are displayed in Figure 6, and the coherence sizes are 5.8(r), 3.2(i), and 5.6(a). Comparing these coherence sizes we see that $L_{\mathrm{f}}^{(i)} \approx L_{\mathrm{f}}^{(a)} \approx L_{\mathrm{f}} / 2$. The relation $L_{\mathrm{f}}^{(a)} \approx L_{\mathrm{f}} / 2$ is natural since $G^{2}$ should decay approximately twice faster than $G$, which allows us to choose $L_{\mathrm{f}}^{(a)}$ as the coherence length related to $G_{m n}{ }^{2}(\epsilon)$ and treat $L_{\mathrm{f}}^{(a)} \approx L_{\mathrm{f}} / 2$ as the effective coherence size $L_{\text {eff. }}$ This yields the following approximate expression for the shift in terms of the exciton mean free path:

$$
\Delta \Omega=\frac{4 \pi^{2} J}{L_{\mathrm{eff}}^{2}} \approx \frac{16 \pi^{2} J}{L_{\mathrm{f}}^{2}}
$$

Using $\Delta \Omega=250 \mathrm{~cm}^{-1}$ and an average nearest-neighbor coupling of $\bar{J}=282 \mathrm{~cm}^{-1}$ gives $L_{\mathrm{f}} / 2=6.7$ which is in agreement with $L_{\mathrm{f}} / 2=5.6$ calculated for model III.

In summary we have shown that in all cases the effective coherence size which determines the shift $\Delta \Omega$ of the pumpprobe signal from LH2 is determined by one-half of the exciton mean free path $L_{\mathrm{f}}$ : $L_{\mathrm{eff}} \approx L_{\mathrm{f}} / 2=5.6$, which is induced by exciton-phonon interactions and disorder-induced elastic scattering. This is a very important result since the relation $L_{\mathrm{eff}} \approx$ $L_{\mathrm{f}} / 2$ is valid for quite different physical situations, e.g., in model I there is no exciton localization and the signal can be interpreted in terms of $L_{\mathrm{f}}$ only, whereas in model III excitons are strongly localized and one may interpret $\Delta \Omega$ in terms of either $L_{\mathrm{f}}$ or $l_{\mathrm{A}}$. To resolve this ambiguity, we consider a situation with static disorder and without exciton-phonon coupling, where the signals can be interpreted in terms of exciton states. It follows from eq 3 that the mean free path $L_{\mathrm{f}}$ associated with $G_{m n}(\omega)$ reflects the properties of both phase and amplitude of the exciton wave functions, whereas the Anderson localization length $l_{\mathrm{A}}$ describes properties of the amplitude only. In higher dimensional systems with weak disorder, there is no exciton localization and $L_{\mathrm{f}}$ is determined by loss of exciton phase (elastic scattering). In one-dimensional systems all exciton states are localized, and since at the band edge the wave functions are nonoscillatory we have the opposite situation whereby $L_{\mathrm{f}}$ reflects localization of amplitude, described by $l_{\mathrm{A}}$.

In summary, our analysis shows that the shift $\Delta \Omega$ is directly related to $L_{\mathrm{eff}} \approx L_{\mathrm{f}} / 2$. Since exciton localization is only one of several mechanisms that determine $L_{\mathrm{f}}$, it is not generally possible to infer that the shift $\Delta \Omega$ is evidence of exciton localization.

\section{Density Matrix Representation of the Exciton Localization Size}

We have shown in previous sections that exciton dephasing and localization show up in a similar way in the pump-probe peak shift $\Delta \Omega$. To distinguish between dephasing and localization, one needs to consider an observable which is influenced in a different way by these two mechanisms. The simplest such quantity is the single-exciton density matrix $N_{m n}$. In the next section we demonstrate how $N_{m n}$ shows up in cooperative spontaneous emission superradiance. A detailed study of the role of $N_{m n}$ in time-resolved emission is given in ref 48 . 

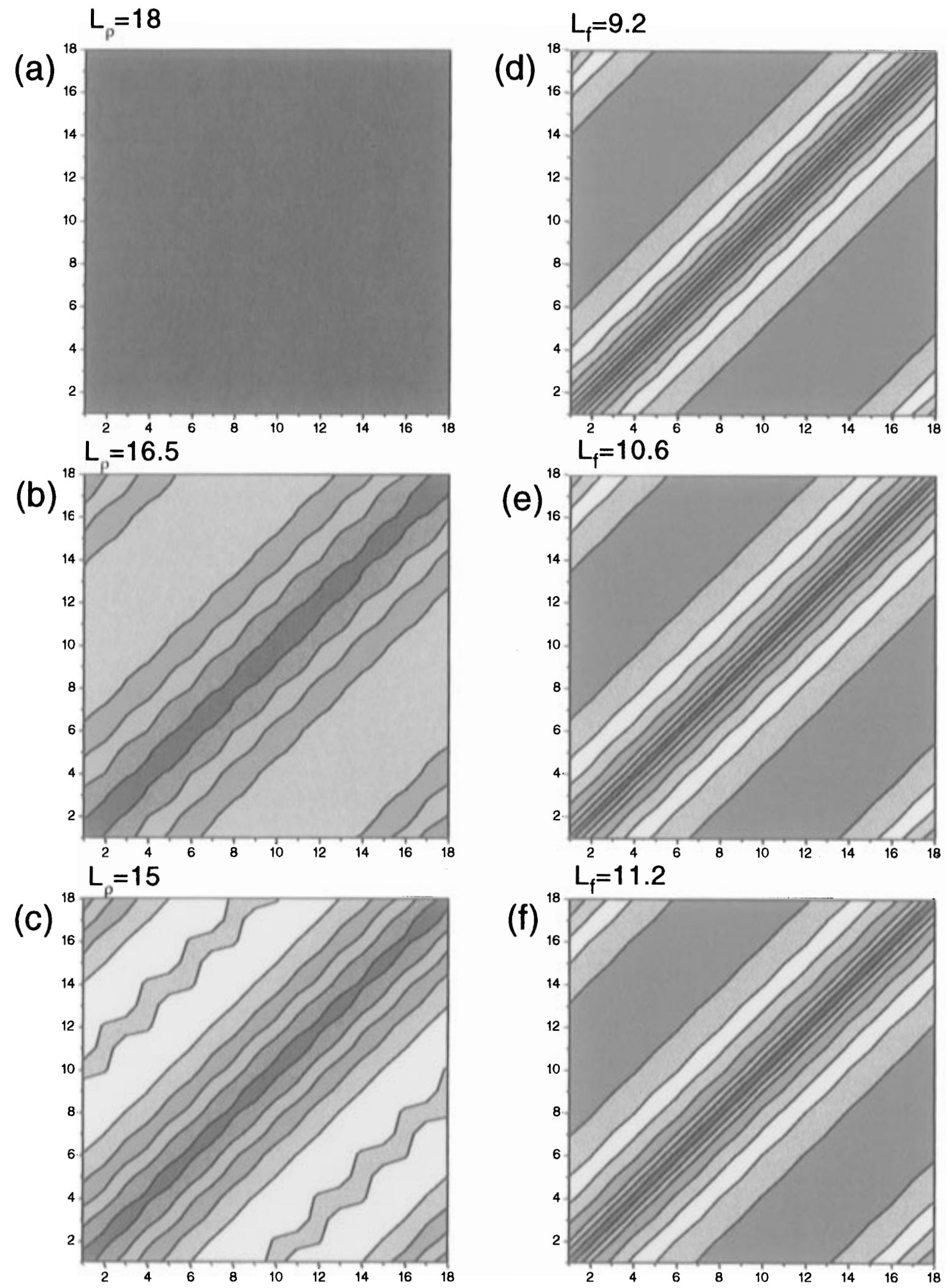

Figure 5. Absolute values of equilibrium exciton density matrixes $N_{m n}$ for models I (a), II (b), and III (c) at $T=4.2 \mathrm{~K}$. Absolute value of the exciton Green function $G_{m n}(\epsilon)$ with $\epsilon$ at the absorption maximum for models I (d), II (e), and III (f). Also given are the corresponding values of the coherence sizes $L_{\rho}$ and $L_{\mathrm{f}}$ calculated via the inverse participation ratio. From large to small: blue, green, yellow, red; see color code in Figure 7.

To determine the role of exciton localization, we display the exciton reduced density matrix $N_{m n}=\left\langle B_{n}^{\dagger} B_{m}\right\rangle$, where $B^{\dagger}{ }_{n}\left(B_{m}\right)$ are the exciton creation (annihilation) operators in the molecular representation. $^{2}$ [Note that we are considering here a nonstationary density matrix whose time evolution is governed by the molecular Hamiltonian driven by the pump field. The localization size can be alternatively obtained using the equilibrium correlation function $\left\langle B_{m}(0) B_{n}^{\dagger}(t) B_{n}(t) B_{m}^{\dagger}(0)\right\rangle$. Here the time evolution of all operators is given by the molecular Hamiltonian (without the external field). The localization length then shows up if we consider this quantity as a function of $n-m$ for long times (or for small frequencies, in the frequency domain).]

The exciton density matrix provides a natural measure of exciton-localization signatures of optical measurements such as 

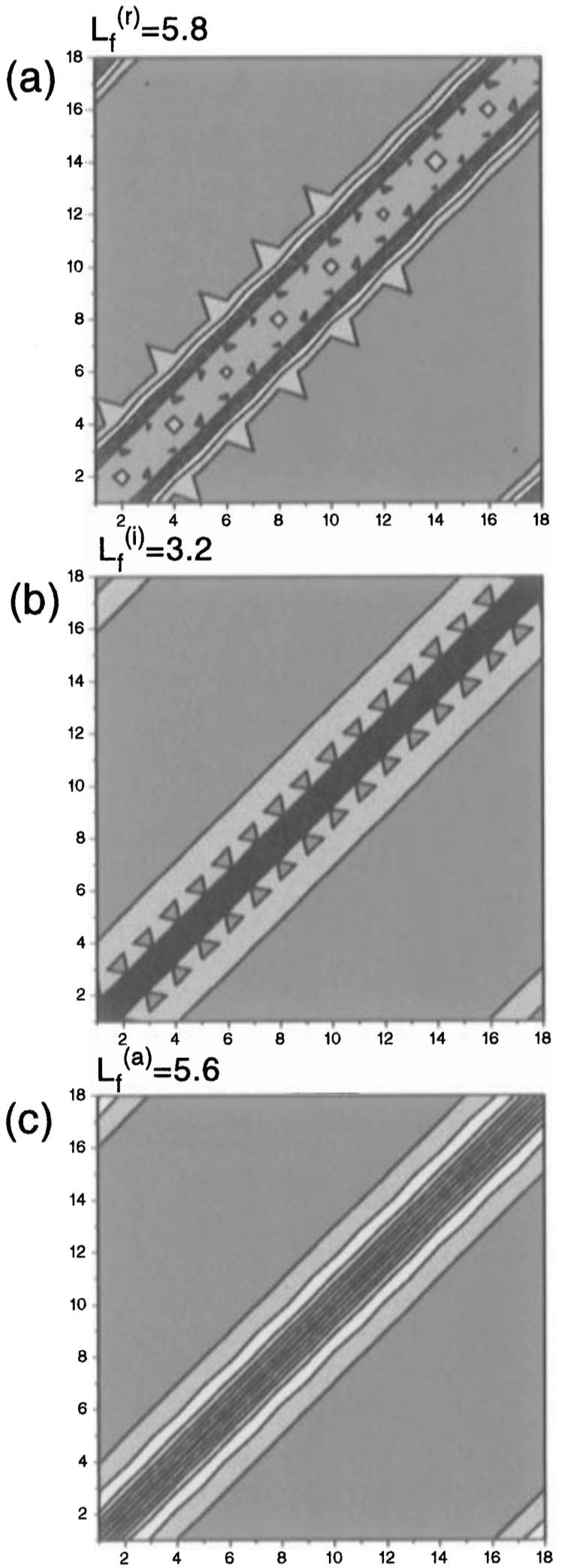

Figure 6. Square of exciton Green function $G_{m n}{ }^{2}(\epsilon)$ with $\epsilon$ at the absorption maximum for model III. Absolute value of real part (a), absolute value of imaginary part (b), and absolute value (c). Also given are the corresponding values of the coherence sizes $L_{\mathrm{f}}^{(r)}, L_{\mathrm{f}}^{(i)}$, and $L_{\mathrm{f}}^{(a)}$ calculated via the inverse participation ratio. From large to small: blue, green, yellow, red; see color code in Figure 7.

pump-probe spectroscopy. When the exciton eigenstates are well resolved and the experiment depends on a few of them, it is possible to interpret measurements using properties of individual eigenstates. However, in many cases, these properties are averaged out by the collective nature of the measurement.
This happens, e.g., at finite temperatures (compared with the exciton bandwidth). In addition, when exciton-phonon coupling is incorporated, the pure exciton states are not very meaningful, since we need to consider the eigenstates in the joint electronic and nuclear space. The density matrix provides the level of description most compatible with optical measurements and is therefore the natural link between theory and experiment. In section VI we show that the localization of the density matrix as viewed by its "antidiagonal" sections controls the superradiance emission, regardless of whether it represents a few individual eigenstates or the collective effect of many states.

Neglecting disorder, the density matrices representing the lowest exciton states $\left\langle\alpha\left|B_{n}^{\dagger} B_{m}\right| \alpha\right\rangle, \alpha=1,2,3$ are displayed in Figures 7 and 5a. The lowest optically forbidden exciton (Figure 5a) is completely delocalized over the entire ring, and all density matrix elements are the same. The next two optically active excitons are degenerate with an energy $60 \mathrm{~cm}^{-1}$ higher than the lowest exciton. These excitons are somewhat localized, as shown in Figure 7a,b. The density matrix representing a mixed state, with both excitons equally populated displayed in Figure 7c, shows a cyclic symmetry. The modulation of the offdiagonal elements reflects the weak dimerization.

A convenient measure of exciton delocalization is provided by the inverse participation ratio of the exciton density matrix

$$
L_{\rho} \equiv\left[L \sum_{m n}\left|\rho_{m n}\right|^{2}\right]^{-1}\left[\left(\sum_{m n}\left|\rho_{m n}\right|\right)^{2}\right]
$$

with $\rho_{m n}=N_{m n}$. Equation 15 is the same formula used for the definition of $L_{\mathrm{f}}$ (see eq 12), with $G_{m n}(\epsilon)$ replaced by $\rho_{m n}$. This quantity gives the length scale on which the density matrix decays along the "antidiagonal" direction, i.e., as a function of $n-m$. Similar measures has been successfully used in the analysis of off resonant polarizabilities of aggregates, ${ }^{42,45}$ conjugated polymers, ${ }^{46}$ and semiconductor nanocrystals. ${ }^{47}$

Figure 7 displays the thermally equilibrated density matrixes for model I corresponding to $100 \mathrm{~K}$ (Figure d) and $300 \mathrm{~K}$ (Figure e). Although the individual eigenstates are delocalized, the thermal exciton density matrix is localized. This thermal localization increases with increasing temperatures, from $L_{\rho}=$ 13.8 at $100 \mathrm{~K}$ to $L_{\rho}=7.9$ at $300 \mathrm{~K}$. At higher temperatures when all excitons are populated equally, the density matrix becomes diagonal and $L_{\rho}=1$.

The present definition of a localization size is in general different from that based on the size of individual eigenstates. If the eigenstates are localized, then the density matrix will be localized as well. As demonstrated in Figure 5, the reverse is not true: the density matrix may be localized even if the individual states are delocalized. Since optical measurements are related to the density matrix, we argue that this new definition is more appropriate. It provides precisely the level of averaging required for the description of optical signals.

Figure $5 \mathrm{a}-\mathrm{c}$ shows the equilibrium density matrixes corresponding to models I, II, and III at $4.2 \mathrm{~K}$. For model I, the lowest exciton, which is the only one populated at $T=4.2 \mathrm{~K}$, is delocalized over the entire aggregate. In this coherent case, $L_{\rho}=18$ coincides with the systems' physical size. In the opposite, incoherent case, when the density matrix is completely diagonal in real-space, we have $L_{\rho}=1$. The disorder included in models II and III leads to a decrease of $L_{\rho}$ to 16.5 and 15.0, which reflects effects of exciton localization on the exciton coherence size. It is important to note that in all cases excitonphonon interaction has been taken into account in the weak coupling limit which results in Boltzmann distribution of excitons at large times. This allows to interpret our results in 

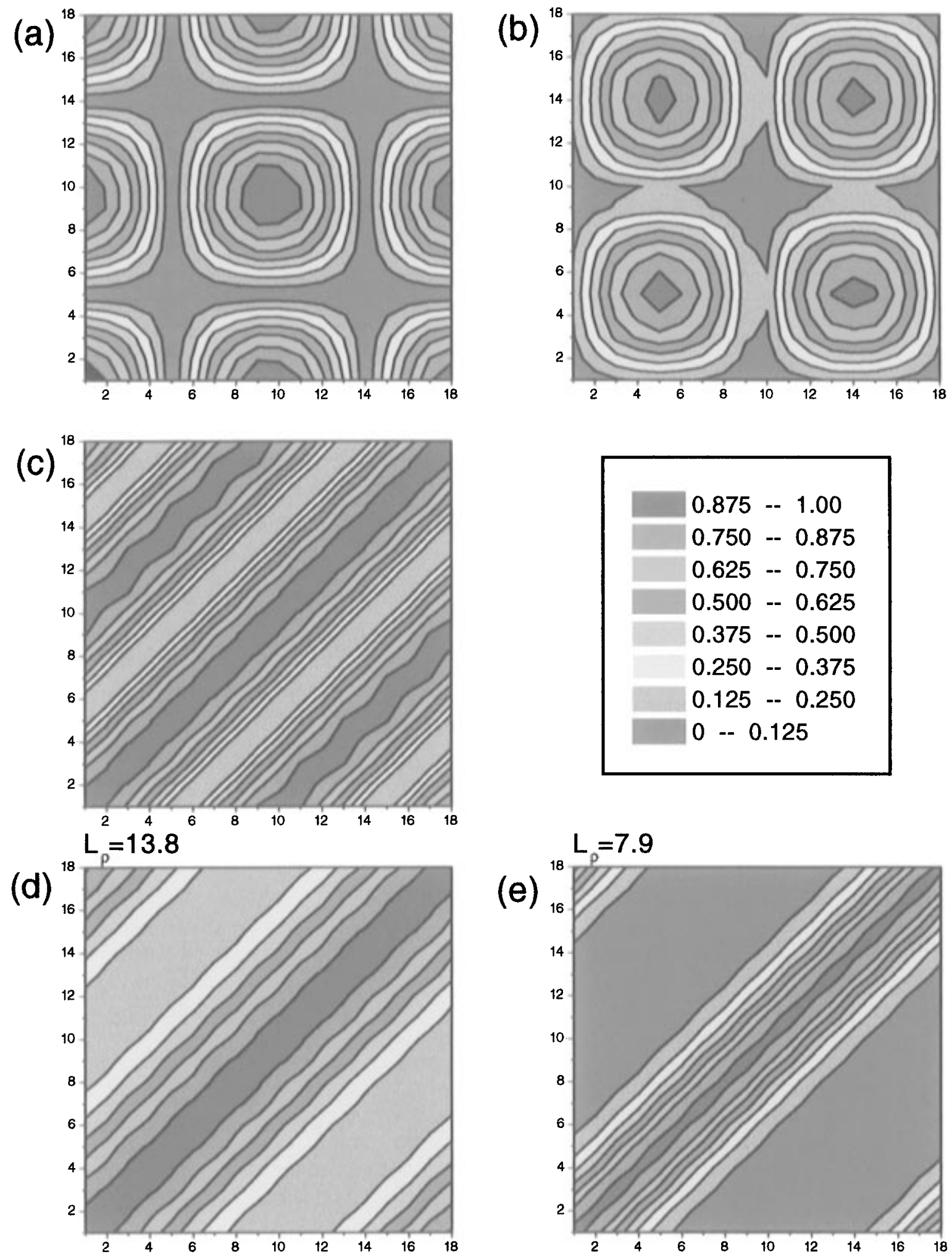

Figure 7. $(\mathrm{a}, \mathrm{b})$ Absolute values of density matrixes of the two lowest degenerate optically active excitons of B850 of LH2 without disorder (model I). (c) Mixed state with equal populations of states (a) and (b). Also shown are the absolute values of equilibrium exciton density matrixes $N_{m n}$ at $100 \mathrm{~K}$ (d) and $300 \mathrm{~K}$ (e) and the corresponding values of the coherence size $L_{\rho}$ calculated via the inverse participation ratio are given. From large to small: blue, green, yellow, red; see color code.

the following way: weak exciton-phonon interaction does not lead to localization of the density matrix $\left(L_{\rho}=18\right)$ whereas disorder does $\left(L_{\rho}<18\right)$. Since at low temperatures only lowenergy excitons with nonoscillatory wave functions are populated, $L_{\rho}$ is related to the exciton localization length $l_{\mathrm{A}}$ but not to $L_{\mathrm{f}}$ since the phonon-induced part of $L_{\mathrm{f}}$ does not affect to $L_{\rho}$ at all $\left(L_{\rho}=L\right.$ for model $\left.\mathrm{I}\right) . l_{\mathrm{A}}$ is usually defined using the participation ratio of the exciton wave function. ${ }^{44,48}$ Even when
$L_{\rho}$ is determined by localization, its numerical value does not coincide with $l_{\mathrm{A}}$. For the values of parameters used in this paper $L_{\rho} \approx 3 l_{\mathrm{A}} \cdot{ }^{48}$ This means that for model III $\left(L_{\rho}=15\right)$ excitons are localized $\left(l_{\mathrm{A}}=5\right)$.

We emphasize that $L_{\rho}$ is a measure of exciton localization only at low temperatures and for weak exciton-phonon coupling. At higher temperatures higher energy excitons with oscillatory wave functions are populated, thereby decreasing $L_{\rho}$. 
Under these conditions exciton localization does not contribute to $L_{\rho}$, which is primarily determined by the size $l_{\mathrm{T}}$ defined in the Introduction. ${ }^{48}$ Strong exciton-phonon coupling can result in another coherence size that affects $L_{\rho}$ : the polaron size $l_{\mathrm{p}}$, with the polaron binding energy $E_{\mathrm{p}}$ being the characteristic energy parameter. ${ }^{49}$

Polaron formation can also contribute to $\Delta \Omega$, and the mechanism is very similar to that of localization. Although a polaron is an electronically delocalized state, due to selftrapping, the different positions are correlated. To put this on a formal basis, we consider the reduced exciton density matrix $N_{m n}$ prepared by the pump. Due to lattice distortion $N_{m n}$ as a function of $m-n$ acquires a coherence length $l_{\mathrm{p}}$. Since the relaxed polaron prepared by the pump has an energy $-E_{\mathrm{p}}$ with respect to the exciton band edge, the energy of a two-exciton state created by the probe is shifted by at least $E_{\mathrm{p}}$ with respect to twice the energy of a single exciton. This makes $E_{\mathrm{p}}$ contribution to $\Delta \Omega$. Taking polaron effects into account is a natural extension of the present theory. The energy parameters related to the coherence sizes $l_{\mathrm{ph}}, l_{\mathrm{p}}$, and $l_{\mathrm{A}}$ are the homogeneous line width $2 \Gamma_{\mathrm{ph}}$, the polaron binding energy $E_{\mathrm{p}}$, and the minimal energy splitting between overlapping localized exciton states $\Delta E_{\mathrm{a}}$, respectively. For each of these three mechanisms the splitting between the peaks $\Delta \Omega$ may be attributed to one of the corresponding energy parameters.

\section{Superradiance Coherence Size}

Cooperative spontaneous emission (superradiance) provides an additional interesting signature of exciton localization. When molecules radiate in phase, we expect the radiative decay rate to be enhanced. The superradiance coherence size $L_{\mathrm{s}}$ is defined as the ratio of the radiative decay rate of the aggregate to that of a single molecule. ${ }^{50}$ It reflects the extent of excitonic intermolecular coherence. Earlier calculations have addressed the role of exciton-phonon coupling and disorder on $L_{\mathrm{s}} .{ }^{50}$ The superradiance enhancement factor $L_{\mathrm{s}}^{50}$ can be expressed in terms of the exciton density matrix: ${ }^{48}$

$$
L_{\mathrm{s}}(t)=\sum_{m n}\left(\mathbf{d}_{m} \cdot \mathbf{d}_{n}\right) N_{m n}(t)
$$

Here the matrix $N_{m n}$ is normalized to have a unit trace, i.e., $\sum_{n} N_{n n}=1$. The direction of the molecular dipole moments is given by the unit vectors $\mathbf{d}_{m}$. Unlike $\Delta \Omega$, superradiance is directly related to the exciton localization size. Initially, following the excitation, $L_{\mathrm{s}}(t)$ will vary with time. Below we consider the long time limit where the density matrix has been fully equilibrated in the exciton manifold. Temperaturedependent superradiance have been recently reported in LH1 and LH2. ${ }^{28}$ The predicted superradiance enhancement should provide a critical test for our model, since all parameters have been already determined.

In Figure 8 we display the superradiance enhancement factor $L_{\mathrm{s}}$ for models I, II, and III. In the absence of disorder (model I) and at low temperatures only the lowest exciton state is occupied. Although this exciton and the corresponding density matrix are delocalized over the entire system (Figure 5a), the enhancement factor $L_{\mathrm{s}}$ is zero. This is due to the fact that given the geometry of LH2 with a tangential head-to-tail orientation of the dipoles, the lowest exciton carries no oscillator strength. With increasing temperature, higher bright exciton states are populated, and $L_{\mathrm{s}}$ is finite. The density matrix, however, becomes more localized as the temperature is increased. ${ }^{48}$ The increase of $L_{\mathrm{s}}$ as the coherence size of the density matrix $L_{\rho}$

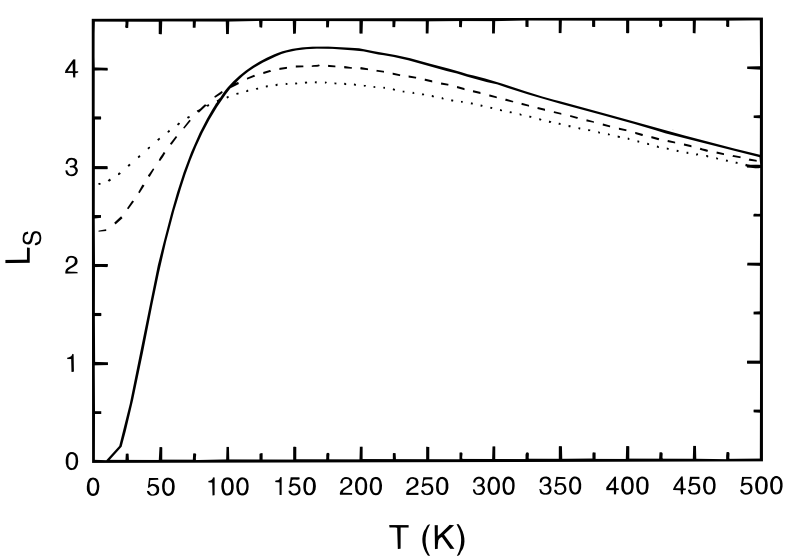

Figure 8. Superradiance enhancement factor $L_{\mathrm{S}}$ as function of temperature for models I (solid), II (dashed), and III (dotted).

decreases, suggests that in the low-temperature regime $L_{\mathrm{S}}$ is related directly to the difference between the physical site $L$ and $L_{\rho}$. At $T=170 \mathrm{~K}$ corresponding to a thermal energy of $107 \mathrm{~cm}^{-1}$, which is somewhat larger than the energy difference of $60 \mathrm{~cm}^{-1}$ between the lowest and the optically active excitons, $L_{\mathrm{s}}$ reaches a maximum of 4.2 for model I. For higher temperatures $L_{\mathrm{s}}$ decreases. For temperatures much higher than the bandwidth (in model I the bandwidth is $1160 \mathrm{~cm}^{-1}$ corresponding to a temperature of $1850 \mathrm{~K}$ ) all excitons are equally populated. In this case the density matrix is diagonal and $L_{\mathrm{s}}$ becomes 1 .

When disorder is introduced, the pronounced temperature dependence of $L_{\mathrm{s}}$ is diminished. In particular at very low temperatures $L_{\mathrm{s}}$ attains a finite value, since with disorder the transition to the lowest exciton becomes allowed. At $4.2 \mathrm{~K}$ for model II we have $L_{\mathrm{s}}=2.5$ and $L_{\rho}=16.6$. $L_{\mathrm{s}}$ is thus almost exactly given by $L-L_{\rho}=2.4$. For model III we find $L_{\mathrm{s}}=2.8$ and $L_{\rho}=15$, i.e., $L-L_{\rho}=3$. This again demonstrates that at low temperatures $L_{\mathrm{s}}$ is determined by the disorder-induced loss of coherence of the density matrix. With disorder, a smaller maximum of $L_{\mathrm{s}}$ appears around $T=170 \mathrm{~K}$ (4.0 for model II and 3.9 for model III) compared with model I. At higher temperatures $L_{\mathrm{s}}$ decreases in all models, and for very high temperatures, when all excitons are populated equally, it approaches a value of 1 . Our calculations for model III are in good agreement with recent experiments, ${ }^{28}$ which found between 4 and $300 \mathrm{~K}$ a constant value of 2.8 for the superradiance enhancement factor in LH2. Model III reproduces the experimental value for low temperatures and shows a rather weak temperature dependence of $L_{\mathrm{s}}$.

It is important to note that for model III $l_{\mathrm{A}} \approx 5$ (see ref 48 ) and the superradiant factor $L_{\mathrm{s}} \approx 3$ may seem to be formed by coherent emission of dipoles on the length scale of $l_{\mathrm{A}}$. However, this is a coincidence, since we have shown that the size on which dipoles emit coherently is $L_{\rho} \approx 3 l_{\mathrm{A}}$ and for the present geometry $L_{\mathrm{s}} \approx L-L_{\rho} \approx L-3 l_{\mathrm{A}}$ which turns out to be not very different from $l_{\mathrm{A}}$ for $L=18$. For this reason, the different superradiance factor observed in LH1 $\left(L_{\mathrm{s}} \approx 9 \text { with } L=32\right)^{28}$ does not necessarily imply a different exciton coherence size.

Acknowledgment. We wish to thank G. R. Fleming, R. van Grondelle, V. Sundström, and G. J. Small for useful discussions. The support of the Air Force office of scientific research, the National Science Foundation Center for Photoinduced Charge Transfer, and the National Science Foundation through Grants No. CHE-9526125 and No. PHY94-15583 is gratefully acknowledged. T.M. acknowledges financial support by a fellowship from the scientific branch of the NATO through the Deutscher Akademischer Austauschdienst (DAAD). 


\section{References and Notes}

(1) Van Grondelle, R.; Dekker, J. P.; Gillbro, T.; Sundström, V. Biochim. Biophys. Acta 1994, 1187, 1.

(2) Sundström, V.; van Grondelle, R. In Anoxygenic Photosynthetic Bacteria; Blankenship, R. E., Madiga, M. T., Baner, C. E., Eds.; Kluwer Academic: Dordrecht, 1995; p 349.

(3) McDermott, G.; Prince, S. M.; Freer, A. A.; HawthornthwaiteLawless, A. M.; Papiz, M. Z.; Cogdell, R. J.; Isaacs, N. W. Nature 1995, 374, 517.

(4) Sauer, K.; Cogdell, R. J.; Prince, S. M.; Freer, A. A.; Isaacs, N. W.; Scheer, H. Photochem. Photobiol. 1996, 64, 564.

(5) Alden, R. G.; Johnson, E.; Nagarajan, V.; Parson, W. W.; Law, C. J.; Cogdell, R. G. J. Phys. Chem. B 1997, 101, 4667.

(6) De Caro, C.; Visschers, R. W.; van Grondelle, R.; Völker, S. J. Phys. Chem. 1994, 98, 10584.

(7) Jimenez, R.; Dikshit, S. N.; Bradforth, S. E.; Fleming, G. R. J. Phys. Chem. 1996, 100, 6825.

(8) Joo, T.; Jia, Y.; Yu, J.-Y.; Jonas, D. M.; Fleming, G. R. J. Phys. Chem. 1996, 100, 2399.

(9) Reddy, N. R. S.; Small, G. J.; Seibert, M.; Picorel, R. Chem. Phys. Lett. 1991, 181, 391. 9458.

(10) Reddy, N. R. S.; Picorel, R.; Small, G. J. J. Phys. Chem. 1992, 96,

(11) Pullerits, T.; Chachisvilis, M.; Sundström, V. J. Phys. Chem. 1996, $100,10787$.

(12) Hess, S.; Feldchtein, F.; Babin, A.; Nurgaleev, I.; Pullerits, T.; Sergeev, A.; Sundström, V. Chem. Phys. Lett. 1993, 216 , 247.

(13) Monshouwer, R.; de Zarate, I. O.; van Mourik, F.; van Grondelle, R. Chem. Phys. Lett. 1995, 246, 341 .

(14) Chachisvilis, M.; Fidder, H.; Pullerits, T.; Sundström, V. J. Raman Spectrosc. 1995, 26, 513.

(15) Chachisvilis, M. Thesis, Lund University, 1996. Chachisvilis, M.; Pullerits, T.; Westerhuis, W.; Hunter, C. N.; Sundström, V. J. Phys. Chem. $B$ 1997, 101, 7275 .

(16) Nagarajan, V.; Alden, R. G.; Williams, J. C.; Parson, W. W. Proc. Natl. Acad. Sci. U.S.A. 1996, 93, 13774.

(17) Jimenez, R.; van Mourik, F.; Fleming, G. R. J. Phys. Chem. B 1997, 101,7350

(18) Visschers, R. W.; van Mourik, F.; Monshouwer, R.; van Grondelle, R. Biochim. Biophys. Acta 1993, 1141, 238.

(19) Bradforth, S. E.; Jimenez, R.; van Mourik, F.; van Grondelle, R.; Fleming, G. R. J. Phys. Chem. 1995, 99, 16179.

(20) Chachisvilis, M.; Pullerits, T.; Jones, M. R.; Hunter, C. N.; Sundström, V. Chem. Phys. Lett. 1994, 224, 345.

(21) Leupold, D.; Voigt, B.; Pfeiffer, M.; Bandilla, M.; Scheer, H. Photochem. Photobiol. 1993, 57, 24.

(22) Spano, F. C.; Mukamel, S. Phys. Rev. Lett. 1991, 66, 1197; J. Chem. Phys. 1991, 95, 7526.

(23) van Burgel, M.; Wiersma, D. A.; Duppen, K. J. Chem. Phys. 1995, $102,20$.

(24) Loring, R. F.; Mukamel, S. J. Chem. Phys. 1986, 85, 1950 Mukamel, S.; Franchi, D. S.; Loring, R. F. J. Lumin. 1987, 38, 1.

(25) Durrant, J. R.; Knoester, J.; Wiersma, D. A. Chem. Phys. Lett. 1994, 222,450 .
974.

(27) Porter, C. E. Statistical Theory of Spectra: Fluctuations; Academic Press: New York, 1965.

(28) Monshouwer, R.; Abrahamson, M.; van Mourik, F.; van Grondelle, R. J. Phys. Chem. B 1997, 101, 7241.

(29) Chernyak, V.; Wang, N.; Mukamel, S. Phys. Rep. 1995, 263, 213.

(30) Bloembergen, N. Nonlinear Optics; Benjamin: New York, 1965

(31) Kühn, O.; Chernyak, V.; Mukamel, S. J. Chem. Phys. 1996, 105, 8586.

(32) Mukamel, S. Principles of Nonlinear Optical Spectroscopy; Oxford: New York, 1995.

(33) Kadanoff, L. P.; Baym, F. Quantum Statistical Mechanics; Benjamin: New York, 1962.

(34) Lipavsky, P.; Spicka, V.; Velicky, B. Phys. Rev. B 1986, 34, 6933.

(35) Agranovich, V. M.; Konobeev, Y. Sov. Phys. Solid State 1963, 5 , 999. Agranovich, V. M.; Konobeev, Y. Phys. Status Solidi 1965, 27, 435. Kenkre, V. M.; Knox, R. S. Phys. Rev. B 1974, 9, 5279. Silbey, R.; Munn, R. W. J. Chem. Phys. 1980, 72, 2763. Kenkre, V. M.; Reinecker, P. In Exciton Dynamics in Molecular Crystals and Aggregates; Springer Tracts in Modern Physics; Springer: Berlin, 1982.

(36) Chernyak, V.; Mukamel, S. Phys. Status Solidi B 1995, 189, 67.

(37) Chernyak, V.; Mukamel, S. To be published.

(38) Zwanzig, R. Lect. Theory. Phys. 1961, 3, 106. Zwanzig, R. Physica 1964, 30, 1109. Mori, H. Prog. Theor. Phys. 1965, 33, 423. Mori, H. Prog. Theor. Phys. 1965, 4, 399.

(39) Redfield, A. G. Adv. Magn. Reson. 1965, $1,1$.

(40) Leegwater, J. A.; Mukamel, S. Phys. Rev. A 1992, 46, 452.

(41) Knoester, J.; Mukamel, S. Phys. Rep. 1991, 205, 1.

(42) Dubovsky, O.; Mukamel, S. J. Chem. Phys. 1991, 95, 7828.

(43) Angerhofer, A.; Cogdell, R. J.; Hipkins, M. F. Biochim. Biophys. Acta 1986, 848, 1983.

(44) Economou, E. N. Green's Functions in Quantum Physics; Springer: New York, 1983.

(45) Mukamel, S. In Molecular Nonlinear Optics; Zyss, J., Ed.; Academic Press: New York, 1994.

(46) Mukamel, S.; Wang, H. X. Phys. Rev. Lett. 1992, 69, 65. Mukamel, S.; Wang, H. X. In Optics of Semiconductor Nanostructures; Henneberger, F., Schmitt-Rink, S., Göbel, E. O., Eds.; Akademie Verlag: Berlin, 1993. Mukamel, S.; Takahashi, A.; Wang, H. X.; Chen, G. Science 1994, 266 , 250.

(47) Yokojima, S.; Meier, T.; Mukamel, S. J. Chem. Phys. 1997, 106 3837.

(48) Meier, T.; Zhao, Y.; Chernyak, V.; Mukaeml, S. J. Chem. Phys., in press

(49) Rashba, E. I. Opt. Spektrosk. 1957, 2, 75. Holstein, T. Ann. Phys. 1959, 8, 325. Fröhlich, H. Proc. R. Soc. London A 1937, 160, 230; Adv Phys. 1954, 3, 325. Toyozawa, Y. Prog. Theor. Phys. 1961, 26, 29. Merrifield, R. E. J. Chem. Phys. 1964, 40, 445.

(50) Grad, J.; Hernandez, G.; Mukamel, S. Phys. Rev. A 1988, 37, 2835. Spano, F. C.; Mukamel, S. J. Chem. Phys. 1989, 91, 683. Spano, F. C.; Kuklinski, J. R.; Mukamel, S. Phys. Rev. Lett. 1990, 65, 211; J. Chem. Phys. 1991, 94, 7534. 